\title{
Comparison of transverse and modified subtrochanteric femoral shortening osteotomy in total hip arthroplasty for developmental dysplasia of hip: a meta-analysis
}

\author{
Changchuan Li, Chi Zhang, Maolin Zhang and Yue Ding*
}

\begin{abstract}
Background: Subtrochanteric femoral shortening osteotomy is a crucial procedure to prevent nerve injury in total hip arthroplasty for severe developmental dysplasia of the hip. Transverse osteotomy was first applied, and other modified methods have also been reported. Each has its own advantages and limitations, but no definitive conclusions regarding differences in outcomes have been reached to date.
\end{abstract}

Methods: We therefore performed a comprehensive meta-analysis to compare the outcomes of different approaches. 37 studies (795 hips) were included in the final analysis. Meta-analysis, subgroup analysis and meta-regression were performed.

Results: Meta-analysis and subgroup analysis showed no significant difference between transverse and modified method. This is further confirmed by meta-regression. Method of osteotomy was found to be not associated with nonunion rate $(P=0.472)$, as well as other post-operative outcomes including nerve palsy $(P=0.240)$, dislocation $(P=0.735)$, revision $(P=0.653)$ and Harris hip score improvement $(P=0.562)$. In addition, western countries $(P=0.010)$ and duration of follow-up more than 5 years $(P=0.014)$ were associated with higher revision rate.

Conclusions: Transverse osteotomy and modified osteotomy appear to be equivalent in terms of nonunion, safety and efficacy. Transverse osteotomy may be recommended, due to its simplicity and convenience in adjusting the anteversion angle. Well-designed and large-sample-size randomized controlled trials are expected to confirm and update the findings of this analysis.

Keywords: Subtrochanteric femoral shortening osteotomy, Total hip arthroplasty, Developmental dysplasia of the hip, Post-operative outcome, Meta-analysis

\section{Background}

Developmental dysplasia of the hip (DDH), formerly defined as congenital dislocation of the hip, is one of the most common neonatal deformities that may have significant influence on the life quality of patient [1]. Its incidence is estimated to be 3 to 5 per 1000 hips, which is clouded by the absence of definitive diagnostic criteria and the wide range of mild to severe anatomical variations that fall within the spectrum of DDH [2]. Although several options exist including proximal femoral and

\footnotetext{
* Correspondence: profdingyue@163.com

Department of Orthopaedic Surgery, Sun Yat-sen Memorial Hospital, No.107 on Yanjiangxi Road, Yuexiu District, 510120 Guangzhou, Guangdong, China
}

periacetabular osteotomies, total hip arthroplasty (THA) remains the standard treatment in end stage DDH, predominantly Crowe type IV in Crowe classification [3] or high dislocation DDH in Hartofilakidis classification [4], which leads to significant pain and loss of function [5].

Severely dysplastic hips present challenging surgical problems. The formation of a false acetabulum superior to the true acetabulum may lead to the need of leg lengthening, during the operation to get the center of rotation more anatomically $[3,6]$. However, leg lengthening over $3-4 \mathrm{~cm}$ is associated with an increased risk of sciatic nerve injury [7]. Femoral shortening osteotomy has become a standard approach to avoid nerve injury [8]. 
Compared with great trochanter osteotomy, subtrochanteric femoral shortening has been more commonly used, because it has lower nonunion rate of osteotomy [9], and preserves the proximal femoral metaphysis, and thus allows for correction of rotation and the use of an uncemented femoral component [10]. On the other hand, it also provides correction of the excessive anteversion and lateral location of the abductor lever [10-12].

There are various techniques for subtrochanteric femoral shortening osteotomy, in attempt to decrease nonunion of osteotomy, which is one of the most commonly reported complications [6]. Transverse subtrochanteric femoral shortening osteotomy was first performed in THA for DDH patients. Subtrochanteric femur was transversely dissected to remove the excessive length, and the both ends of osteotomy were connected (Figure 1a). Due to the centrosymmetry of intersecting surface, adjustment of the two fragments of transverse osteotomy during the surgery is possible, when the initial alignment is not ideal [13]. This is important for the correction of femoral anteversion, the most common and one of the most important anatomic abnormalities caused by DDH $[6,10,14]$, which is correlated with postoperative hip function [14].

However, the centrosymmetry of intersecting surface in turn increases the rotational instability, which in combination with a smaller surface contact might lead to nonunion of the osteotomy [9]. Step-cut [15], oblique [14], and chevron type [16] subtrochanteric femoral shortening osteotomies, which we categorize as "modified osteotomy", were introduced in attempt to enhance the rotational stability of the osteotomy, and to reduce the risk of rotational instability and non-union [17]. Compared with transverse osteotomy, the osteotomy lines are step-cut, oblique and double-V-shaped, respectively (Figure 1b-1d). So they were expected to have lower nonunion rate of osteotomy. Many researchers have published their results and opinions on this issue, but few of them conducted a head-to-head comparison. Moreover, single clinical trials are often underpowered and lack generalizability [18]. Up to date, no definitive conclusions regarding differences in outcomes have been reached. We therefore systemically searched the literatures currently available, and performed a comprehensive meta-analysis to compare the outcomes of the two methods.

\section{Methods}

\section{Data source}

We searched PubMed, Embase and Cochrane Library up to August, 2014 for literatures which focused on subtrochanteric femoral shortening osteotomy for DDH and specified the method of osteotomy and the outcomes, using the following terms: $((((($ congenital $)$ OR developmental)) AND hip) AND (((dysplasia) OR dislocation) OR dislocations))) AND ((subtrochanteric) AND ((osteotomy) OR osteotomies)) (see Additional file 1). There was no restriction to regions or languages. The computer search was supplemented with manual searches of the reference lists of all retrieved literatures. When there were two or more reports describing the same population, the most recent or complete version was involved.

\section{Study eligibility and selection}

The studies have to meet the following pre-determined inclusion criteria:

1. It investigated the subtrochanteric femoral shortening osteotomy in the surgical treatment of $\mathrm{DDH}$, and short- and long-term outcomes of the surgery.

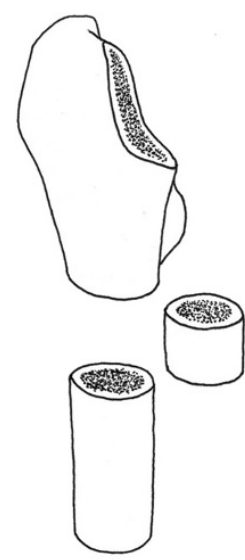

(a)

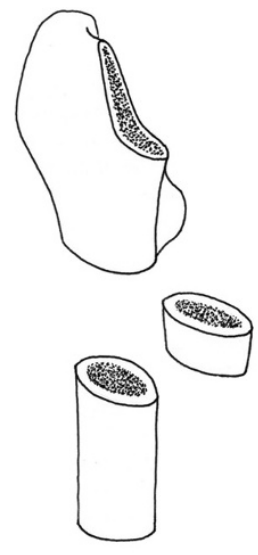

(b)

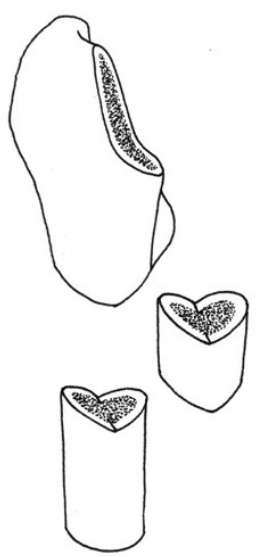

(c)

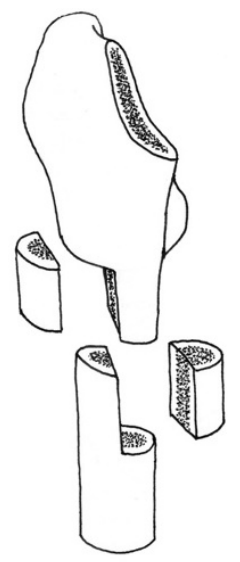

(d)

Figure 1 Schematic illustration of different methods of subtrochanteric femoral shortening osteotomy. (a) transverse (b) oblique (c) double-chevron (d) step-cut. 
2. It provided data that allowed for quantitative analysis.

3. It had been published or accepted for publication.

Literatures that failed to meet the inclusion criteria were excluded. Besides, our exclusion criteria included:

1. Case report or review;

2. Not clinical studies (e.g. biomechanical);

3. Data of interest are not clear, and couldn't be obtained by contacting the authors;

4. Duplicated data.

Two reviewers (C. Li and M. Zhang) independently evaluated the eligibility of involved studies. Discrepancies between evaluators were resolved by discussion or consultation with the corresponding author (Y. Ding).

\section{Data abstraction}

Study characteristics were retrieved including author, year of publication, country, demographics of the population, method of subtrochanteric femoral shortening osteotomy, duration of follow-up, and union of the osteotomy sites. Data were extracted independently and in duplicate by both reviewers (C. Li and C. Zhang). Discrepancies between evaluators were resolved by discussion or consultation with the corresponding author (Y. Ding).

\section{Outcomes of interest}

The following outcomes were used to compare transverse and modified subtrochanteric osteotomy:

\section{Primary outcomes}

1. Nonunion (permanent failure of bone healing without treatment, usually identified at 8 months postoperatively). In consideration that delayed union might also require a revision surgery sometimes, like the case of nonunion, we also included delayed union that required a revision surgery into the category of nonunion.

2. Nerve palsy (transient or permanent, predominantly caused by stretching, could happen to sciatic nerve, femoral nerve, and occasionally to peroneal nerve).

\section{Secondary outcomes}

Dislocation (early or recurrent), revision (due to all causes), leg-length discrepancy (average leg-length discrepancy, and discrepancy within ideal range, which was defined as $0-2 \mathrm{~cm}$ ), Harris hip score (HHS) improvement (proportion of the difference between post- and preoperative HHS in the post-operative HHS) and deep infection.

\section{Statistical analysis}

This meta-analysis was performed according to the recommendations of Preferred Reporting Items for Systematic Reviews and Meta-Analyses [19]. Raw rates of outcome events were calculated in each study. As the inverse variance weight in fixed-effect metaanalysis is suboptimum when dealing with binary data with low probability, the variances of the raw rates were stabilized using Freeman-Tukey double-arcsine transformation [20]. In the meantime, the doublearcsine transformation could avoid the situation where the rate and standard error of a certain outcome is zero, which is not allowed for meta-analysis. All outcomes were reported with 95\% CIs. Wilson's method was used to calculate the $95 \% \mathrm{CI}$ of the estimated rate to construct the forest plot, because the asymptotic method may produce confidence intervals that extend below zero, especially when the rates are estimated to be low.

We estimated heterogeneity between studies with Cochrane's Q (reported as $X^{2}$ and $P$ values), which is calculated as the weighted sum of squared differences between individual study effects and the pooled effect across studies, and the $I^{2}$ statistic, which describes the percentage of variation between studies that is due to heterogeneity rather than chance [21]. $I^{2}$ values of $25 \%, 50 \%$ and $75 \%$ are taken to indicate low, moderate and high degrees of heterogeneity, respectively. When the $I^{2}$ statistic didn't exceed $50 \%$, we selected the fixed effects model, which could achieve higher statistical power than random effects model. Otherwise, random effects model is adopted. Sensitivity analysis was carried out to judge the weight of each study. After the meta-analysis of transformed data, we inversed the pooled estimate and its $95 \%$ CI back to proportions [22]. Up to date, there is no widely accepted scoring system for assessing the methodological quality of observational studies with no control.

Potential sources of heterogeneity were explored further by meta-regression analysis. The factors investigated in meta-regression included method of osteotomy (by comparing transverse and modified osteotomy), country (by comparing western and eastern countries), mean age (as a continuous variable, and then by dichotomizing the studies by the median of 49 years), proportion of female patients (as a continuous variable, and then by dichotomizing the studies by the median of $93 \%$ ), duration of follow-up (as a continuous variable, and then by dichotomizing the studies by the median of 5 years), and year of publication (by dichotomizing the studies by the median of the year 2010). Categorical variables were taken into meta-regression using dummy variables [23].

All analyses were performed using STATA statistical software package (Version 13.0, StataCorp, 2013) using the commands cii (to calculate Wilson CIs), metan (for meta-analysis), metareg (for meta-regression) and 
metabias (to assess the publication bias). Generally, a $P$ value $<0.05$ was considered to indicate statistical significance $(\alpha=0.05)$.

\section{Results}

\section{Characteristics of eligible studies}

37 studies (791 hips) were included in the final analysis (Figure 2). None of them was randomized controlled trial, case-control study or cohort study. The characteristics of the included studies are listed in Table 1. Agreement between the two reviewers was achieved.

Of the 37 studies enrolled in the meta-analysis, 10 were conducted in Europe [11,12,14,15,24-29], 6 in the North America [7,16,30-33], 1 in Australia [34], and the other 20 in Asia [9,13,17,35-51]. Female took the majority of the patients, and 9 studies were based on female patients exclusively.

Most researchers have used only one method of subtrochanteric osteotomy in their own studies, however, with two exceptions. Erdemli et al. [35] applied step-cut osteotomy in 3 hips, and transverse osteotomy in 22 hips. The other researcher, Dallari [14], performed stepcut osteotomy in 14 hips, and oblique osteotomy in 19 hips. These two studies were split in meta-analysis and meta-regression. The transverse group included 24 studies (550 hips), and the modified group included 15 studies (241 hips).

\section{Primary outcomes Nonunion}

Pooled estimate of nonunion rate was 3.79\% (95\% CI 2.60\%-5.20\%). Heterogeneity among the studies was low $\left(I^{2}=14.5 \%\right)$. Data of nonunion were further analyzed in two subgroups (modified osteotomy and transverse osteotomy). Figure 3 showed the forest plot of subgroup analysis. There was no significant difference in nonunion rate between modified group and transverse group (Table 2).

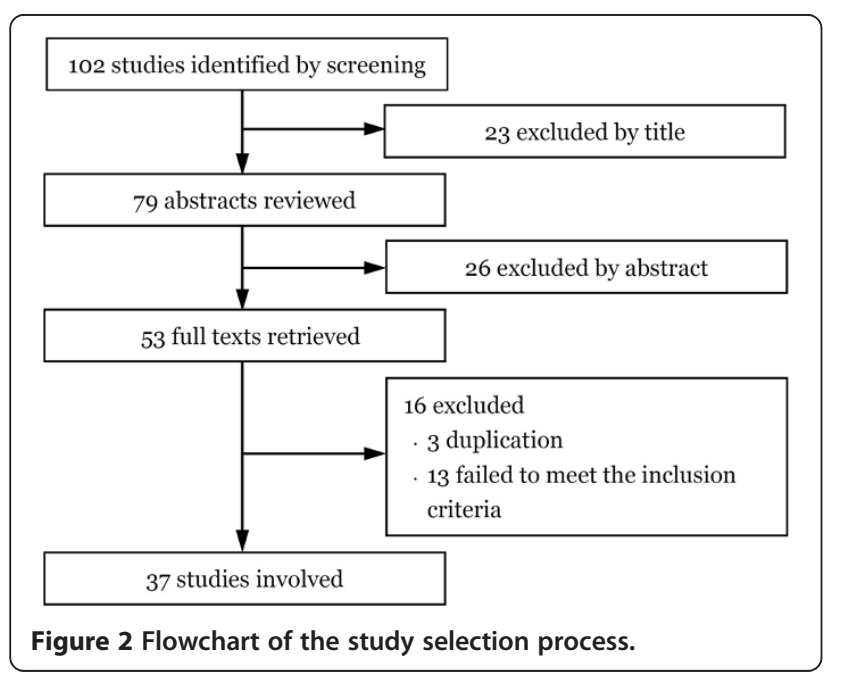

\section{Nerve palsy}

Pooled estimate of nerve palsy rate was $2.63 \%$ (95\% CI $1.60 \%-3.87 \%)$. Forest plot of meta-analysis was shown in Figure 4. According to Table 2, there was so little heterogeneity among the studies $\left(I^{2}<0.1 \%\right)$, that subgroup analysis was not necessary.

\section{Secondary outcomes \\ Dislocation}

Pooled estimate of dislocation rate was 5.88\% (95\% CI $4.22 \%-7.80 \%)$ Heterogeneity among the studies was moderate $\left(I^{2}=36.2 \%\right)$. Data of dislocation were further analyzed in subgroup analysis, and forest plot was constructed (Figure 5). No significant difference in dislocation rate was revealed between modified group and transverse group, and subgroup heterogeneity were not significantly less $\left(I^{2}=38.4 \%\right.$ for modified subgroup and $I^{2}=38.5 \%$ for transverse subgroup) (Table 2 ).

\section{Revision}

Pooled estimate of revision rate due to all causes was 8.90\% (95\% CI 6.56\%-11.50\%). Heterogeneity among the studies was moderate $\left(I^{2}=43.3 \%\right)$. Data of revision were further analyzed in subgroups, and forest plot was constructed (Figure 6). As Table 2 indicated, no significant difference in revision rate was shown between modified group and transverse group.

\section{Leg-length discrepancy}

Only five studies provided related data on the number of patients who achieved ideal rage of leg-length discrepancy. Occurrence of discrepancy out of the ideal range was analyzed. Pooled estimate of occurrence was $6.31 \%$ (95\% CI 2.90\%-11.00\%). Obvious heterogeneity existed among the studies $\left(I^{2}=67.2 \%\right)$. Subgroup analysis was carried out, revealing no significant difference between modified group and transverse group (Table 2).

\section{HHS improvement}

Average HHS in each of the eligible studies have elevated after the surgery. Heterogeneity among the studies was high $\left(I^{2}=96.5 \%\right)$. However, subgroup analysis revealed no significant difference between modified group and transverse group (Table 2).

\section{Deep infection}

Pooled estimate of deep infection rate was $1.34 \%$ (95\% CI $0.46 \%-2.67 \%)$. There was so little heterogeneity among the studies $\left(I^{2}<0.1 \%\right)$, that subgroup analysis was not necessary (Table 2).

\section{Sensitivity analysis}

Sensitivity analysis was carried out by excluding each study in turn to ensure that no single study would be 
Table 1 Characteristics of included studies

\begin{tabular}{|c|c|c|c|c|c|c|c|c|c|}
\hline Study & Country & $\begin{array}{l}\text { Mean age } \\
\text { (range), } \\
\text { year }\end{array}$ & $\begin{array}{l}\text { Sex, } \\
\text { Female \% }\end{array}$ & $\begin{array}{l}\text { Crowe } \\
\text { classification }\end{array}$ & $\begin{array}{l}\text { Total, hips/ } \\
\text { patients }\end{array}$ & $\begin{array}{l}\text { Method of } \\
\text { osteotomy }\end{array}$ & $\begin{array}{l}\text { Mean } \\
\text { follow-up } \\
\text { (range), } \\
\text { years }\end{array}$ & $\begin{array}{l}\text { Nonunion, } \\
\text { hips }\end{array}$ & $\begin{array}{l}\text { Nerve } \\
\text { injury, } \\
\text { hips }\end{array}$ \\
\hline Becker, 1995 & USA & $61(48-72)$ & N/A & IV & $7 / 4$ & Double-chevron & $2.7(0.3-6)$ & 0 & 0 \\
\hline Reikeraas, 1996 & Norway & $54(17-67)$ & 93.8 & IV & $25 / 19$ & Transverse & $(3-7)$ & 0 & 1 \\
\hline Yasgur, 1997 & USA & $42(22-77)$ & 77.8 & IV & $9 / 8$ & Transverse & $3.6(2-7)$ & 1 & 0 \\
\hline Chareancholvanich, 1999 & USA & $51(21-74)$ & N/A & N/A & $15 / 11$ & Double-chevron & $5.5(2-8.5)$ & 0 & 0 \\
\hline Zadeh, 1999 & UK & $49(34-61)$ & 71.4 & I, II \& IV & $7 / 7$ & Transverse & $2.6(1.3-5.0)$ & $1^{*}$ & N/A \\
\hline Bruce, 2000 & Australia & $53(26-77)$ & N/A & III \& IV & $6 / 5$ & Transverse & $4.7(0.5-7.2)$ & 0 & 0 \\
\hline Ozturkmen, 2002 & Turkey & N/A & N/A & IV & $7 / 7$ & Step-cut & N/A & 1 & N/A \\
\hline Sener, 2002 & Turkey & $43(26-64)$ & 95.7 & III \& IV & $28 / 23$ & Step-cut & $4.0(0.6-7.7)$ & 2 & 2 \\
\hline Decking, 2003 & Germany & 47 (19-58) & 70.0 & $\| . \mid I I \& I V$ & $12 / 10$ & Step-cut & $5.1(1.6-10)$ & 0 & 1 \\
\hline Masonis, 2003 & Canada & $48.2(21-70)$ & 84.2 & III \& IV & $21 / 19$ & Transverse & $5.8(2.0-11.2)$ & 2 & 0 \\
\hline \multirow[t]{2}{*}{ Erdemli, 2005} & Turkey & $44(28-61)$ & 100.0 & IV & $25 / 22$ & Step-cut in 3 hips & $5(2-10)$ & 0 & 0 \\
\hline & & & & & & Transverse in 22 hips & & 1 & 0 \\
\hline Bernasek, 2007 & USA & $43(17-67)$ & 91.3 & I.II.|II \& IV & $23 / 20$ & Transverse & $8(5-14)$ & 0 & 0 \\
\hline Gotze, 2007 & Germany & $41.7(29-64)$ & N/A & III \& IV & $7 / 7$ & Transverse & 1.5 & 0 & N/A \\
\hline Makita, 2007 & Japan & $59.6(42-76)$ & 100.0 & IV & $11 / 11$ & Step-cut & $5.4(2.5-14.1)$ & 0 & 2 \\
\hline Park, 2007 & Korea & $44.8(20-66)$ & N/A & III \& IV & $24 / 23$ & Transverse & $4.7(2.0-7.6)$ & 3 & 0 \\
\hline Krych, 2009 & USA & N/A & N/A & IV & $28 / 24$ & Transverse & 4.8 & 2 & 0 \\
\hline Nagoya, 2009 & Japan & $55(44-69)$ & 94.4 & IV & 20/18 & Transverse & $8.1(4-11.5)$ & 0 & 0 \\
\hline Howie, 2010 & UK & $47.3(26-75)$ & N/A & III \& IV & $35 / 28$ & Transverse & $5.6(2-14)$ & 1 & 2 \\
\hline Reikeraas, 2010 & Norway & $48(16-79)$ & 70.8 & III \& IV & $65 / 46$ & Transverse & $13(8-18)$ & 2 & 2 \\
\hline Togrul, 2010 & Turkey & $42.3(33-52)$ & 85.7 & III \& IV & $21 / 14$ & Transverse & $3.4(2.0-5.3)$ & 0 & 0 \\
\hline Akiyama, 2011 & Japan & $58.9(42-77)$ & 90.9 & III \& IV & 15/11 & Transverse & $6.3(2.8-10.4)$ & 3 & 0 \\
\hline Charity, 2011 & UK & $51(33-75)$ & 100.0 & IV & $18 / 15$ & Transverse & $9.5(4.3-14)$ & 1 & 1 \\
\hline \multirow[t]{2}{*}{ Dallari, 2011} & Italy & $52(34-66)$ & 76.9 & IV & $33 / 26$ & Step-cut in 14 hips & $7.3(2.2-15.3)$ & 0 & N/A \\
\hline & & & & & & Oblique in 19 hips & & 1 & N/A \\
\hline Kawai, 2011 & Japan & $64.8(57-73)$ & 100 & IV & $19 / 12$ & Transverse & $3.2(0.5-8)$ & 0 & 0 \\
\hline Kilicarslan, 2011 & Turkey & $46(20-72)$ & N/A & III \& IV & $45 / 31$ & Transverse & $7.2(2.0-10.1)$ & 5 & 0 \\
\hline Starker, 2011 & Germany & 44.6 & 83.3 & IV & $25 / 20$ & Step-cut & N/A & 0 & N/A \\
\hline Takao, 2011 & Japan & 60 & 92.0 & IV & $33 / 25$ & Step-cut & $8(5-11)$ & 0 & 0 \\
\hline Zhong, 2011 & China & $45.2(36-56)$ & 100.0 & IV & $36 / 28$ & Transverse & $4.4(2.3-7.8)$ & 0 & 0 \\
\hline Baz, 2012 & Turkey & $41.6(24-56)$ & 86.7 & IV & $21 / 15$ & Transverse & $4.9(3-8)$ & 0 & 1 \\
\hline Hasegawa, 2012 & Japan & $58.5(48-72)$ & 100.0 & IV & $15 / \mathrm{N} / \mathrm{A}$ & Step-cut & $10.2(5-20)$ & 0 & 1 \\
\hline Semenowicz, 2012 & Poland & 53.4 & 100.0 & IV & $10 / 10$ & Step-cut & 2.3 & 0 & 0 \\
\hline Kilicoglu, 2013 & Turkey & $43(27-60)$ & 95.0 & IV & $20 / 16$ & Oblique & $6.8(3.7-10.3)$ & $1^{*}$ & 0 \\
\hline Li WB, 2013 & China & N/A & 100 & IV & $5 / 5$ & Transverse & N/A & 0 & 0 \\
\hline Li YW, 2013 & China & $54(41-75)$ & 83.3 & IV & $22 / 18$ & Double-chevron & $8(3-12)$ & 0 & 0 \\
\hline Oe, 2013 & Japa & $64.9(35-80)$ & 96.2 & IV & $34 / 26$ & Transverse & $5.2(3-10)$ & 0 & 0 \\
\hline Sun, 2013 & China & $47(38-65)$ & 63.3 & IV & $32 / 27$ & Transverse & $4(0.6-7)$ & 0 & 1 \\
\hline Oinuma, 2014 & Japan & $61.5(46-73)$ & 100 & IV & $12 / 9$ & Transverse & $3.7(1.5-6.3)$ & 0 & 0 \\
\hline Total & / & 50.0 & 88.1 & / & 791/N/A & / & / & 27 & 14 \\
\hline
\end{tabular}




\begin{tabular}{|c|c|c|c|c|c|c|c|c|}
\hline \multirow[t]{2}{*}{ Study } & \multirow[t]{2}{*}{ Revision } & \multirow[t]{2}{*}{ Dislocation } & \multirow{2}{*}{$\begin{array}{l}\text { Deep } \\
\text { infection }\end{array}$} & \multicolumn{2}{|l|}{ Harris hip score } & \multicolumn{2}{|c|}{ Limb-length discrepancy } & \multirow{2}{*}{$\begin{array}{l}\text { Acceptable } \\
\text { discrepancy } \\
\text { patients }\end{array}$} \\
\hline & & & & Pre-operative & Post-operative & Pre-operative & Post-operative & \\
\hline Becker, 1995 & 1 & 0 & $\mathrm{~N} / \mathrm{A}$ & $\mathrm{N} / \mathrm{A}$ & $\mathrm{N} / \mathrm{A}$ & N/A & N/A & $\mathrm{N} / \mathrm{A}$ \\
\hline Reikeraas, 1996 & N/A & N/A & N/A & 43 & 93 & N/A & N/A & N/A \\
\hline Yasgur, 1997 & 1 & 1 & $\mathrm{~N} / \mathrm{A}$ & N/A & N/A & N/A & 1.5 & N/A \\
\hline Chareancholvanich, 1999 & $\mathrm{~N} / \mathrm{A}$ & 0 & N/A & N/A & N/A & 3.9 & 1.4 & N/A \\
\hline Zadeh, 1999 & N/A & N/A & N/A & 44 & 91 & N/A & N/A & $\mathrm{N} / \mathrm{A}$ \\
\hline Bruce, 2000 & N/A & N/A & 0 & 31 & 81 & N/A & N/A & N/A \\
\hline Ozturkmen, 2002 & N/A & N/A & N/A & N/A & N/A & N/A & N/A & N/A \\
\hline Sener, 2002 & 0 & 0 & 0 & 36.9 & 95.3 & N/A & N/A & 19 \\
\hline Decking, 2003 & 1 & 0 & 0 & 36 & 82 & 5.4 & 1.3 & N/A \\
\hline Masonis, 2003 & 3 & 3 & 0 & 32.5 & 73.6 & N/A & N/A & N/A \\
\hline \multirow[t]{2}{*}{ Erdemli, 2005} & N/A & N/A & 0 & N/A & N/A & N/A & N/A & N/A \\
\hline & N/A & N/A & 0 & N/A & N/A & N/A & N/A & N/A \\
\hline Bernasek, 2007 & 1 & 4 & 0 & 42 & 82 & N/A & N/A & N/A \\
\hline Gotze, 2007 & N/A & $\mathrm{N} / \mathrm{A}$ & N/A & 43 & 77 & N/A & N/A & $\mathrm{N} / \mathrm{A}$ \\
\hline Makita, 2007 & 1 & 1 & 0 & N/A & N/A & 4.7 & 1.2 & 7 \\
\hline Park, 2007 & 1 & 1 & N/A & 35.6 & 81.7 & N/A & N/A & 21 \\
\hline Krych, 2009 & $\mathrm{~N} / \mathrm{A}$ & 4 & N/A & 43 & 89 & N/A & N/A & N/A \\
\hline Nagoya, 2009 & 2 & 0 & 0 & N/A & N/A & N/A & 1.2 & $\mathrm{~N} / \mathrm{A}$ \\
\hline Howie, 2010 & 7 & 3 & 1 & N/A & N/A & N/A & N/A & N/A \\
\hline Reikeraas, 2010 & 11 & 1 & $\mathrm{~N} / \mathrm{A}$ & $\mathrm{N} / \mathrm{A}$ & 87 & N/A & 1.0 & $\mathrm{~N} / \mathrm{A}$ \\
\hline Togrul, 2010 & N/A & 2 & 0 & N/A & N/A & N/A & 0.3 & N/A \\
\hline Akiyama, 2011 & 3 & 2 & N/A & $\mathrm{N} / \mathrm{A}$ & $\mathrm{N} / \mathrm{A}$ & 2.9 & 0.3 & 11 \\
\hline Charity, 2011 & 4 & 0 & N/A & N/A & N/A & N/A & N/A & N/A \\
\hline \multirow[t]{2}{*}{ Dallari, 2011} & N/A & $\mathrm{N} / \mathrm{A}$ & $\mathrm{N} / \mathrm{A}$ & $\mathrm{N} / \mathrm{A}$ & N/A & 7.0 & 3.0 & N/A \\
\hline & N/A & N/A & $\mathrm{N} / \mathrm{A}$ & N/A & N/A & & & N/A \\
\hline Kawai, 2011 & N/A & 0 & 0 & $\mathrm{~N} / \mathrm{A}$ & N/A & N/A & N/A & $\mathrm{N} / \mathrm{A}$ \\
\hline Kilicarslan, 2011 & N/A & 2 & N/A & N/A & N/A & 2.9 & 1.4 & $\mathrm{~N} / \mathrm{A}$ \\
\hline Starker, 2011 & N/A & $\mathrm{N} / \mathrm{A}$ & $\mathrm{N} / \mathrm{A}$ & $\mathrm{N} / \mathrm{A}$ & 90 & $\mathrm{~N} / \mathrm{A}$ & 0.8 & 20 \\
\hline Takao, 2011 & 1 & 2 & N/A & N/A & N/A & 5.1 & 2.8 & $\mathrm{~N} / \mathrm{A}$ \\
\hline Zhong, 2011 & $\mathrm{~N} / \mathrm{A}$ & 0 & 0 & 39 & 87 & 5.7 & 0.6 & N/A \\
\hline Baz, 2012 & 2 & 2 & 0 & 36.2 & 90.8 & N/A & N/A & $\mathrm{N} / \mathrm{A}$ \\
\hline Hasegawa, 2012 & 4 & 3 & 0 & 56 & 85 & 3.8 & 1.4 & N/A \\
\hline Semenowicz, 2012 & N/A & $\mathrm{N} / \mathrm{A}$ & $\mathrm{N} / \mathrm{A}$ & 43.7 & 86 & N/A & N/A & $\mathrm{N} / \mathrm{A}$ \\
\hline Kilicoglu, 2013 & 2 & 3 & N/A & 50 & 83 & N/A & 1.0 & $\mathrm{~N} / \mathrm{A}$ \\
\hline Li WB, 2013 & 0 & 0 & N/A & $\mathrm{N} / \mathrm{A}$ & N/A & $\mathrm{N} / \mathrm{A}$ & N/A & $\mathrm{N} / \mathrm{A}$ \\
\hline Li YW, 2013 & 0 & 0 & 0 & 30 & 91.9 & 2.5 & 1 & 18 \\
\hline Oe, 2013 & 0 & 3 & 0 & $\mathrm{~N} / \mathrm{A}$ & $\mathrm{N} / \mathrm{A}$ & 4.7 & 1.2 & 26 \\
\hline Sun, 2013 & 0 & 0 & 0 & 41.7 & 89.1 & N/A & N/A & N/A \\
\hline Oinuma, 2014 & 0 & 1 & 0 & $\mathrm{~N} / \mathrm{A}$ & $\mathrm{N} / \mathrm{A}$ & $\mathrm{N} / \mathrm{A}$ & N/A & $\mathrm{N} / \mathrm{A}$ \\
\hline Total & 45 & 38 & 1 & / & / & / & / & / \\
\hline
\end{tabular}

N/A: not available.

*Delayed union that needs a second procedure. 


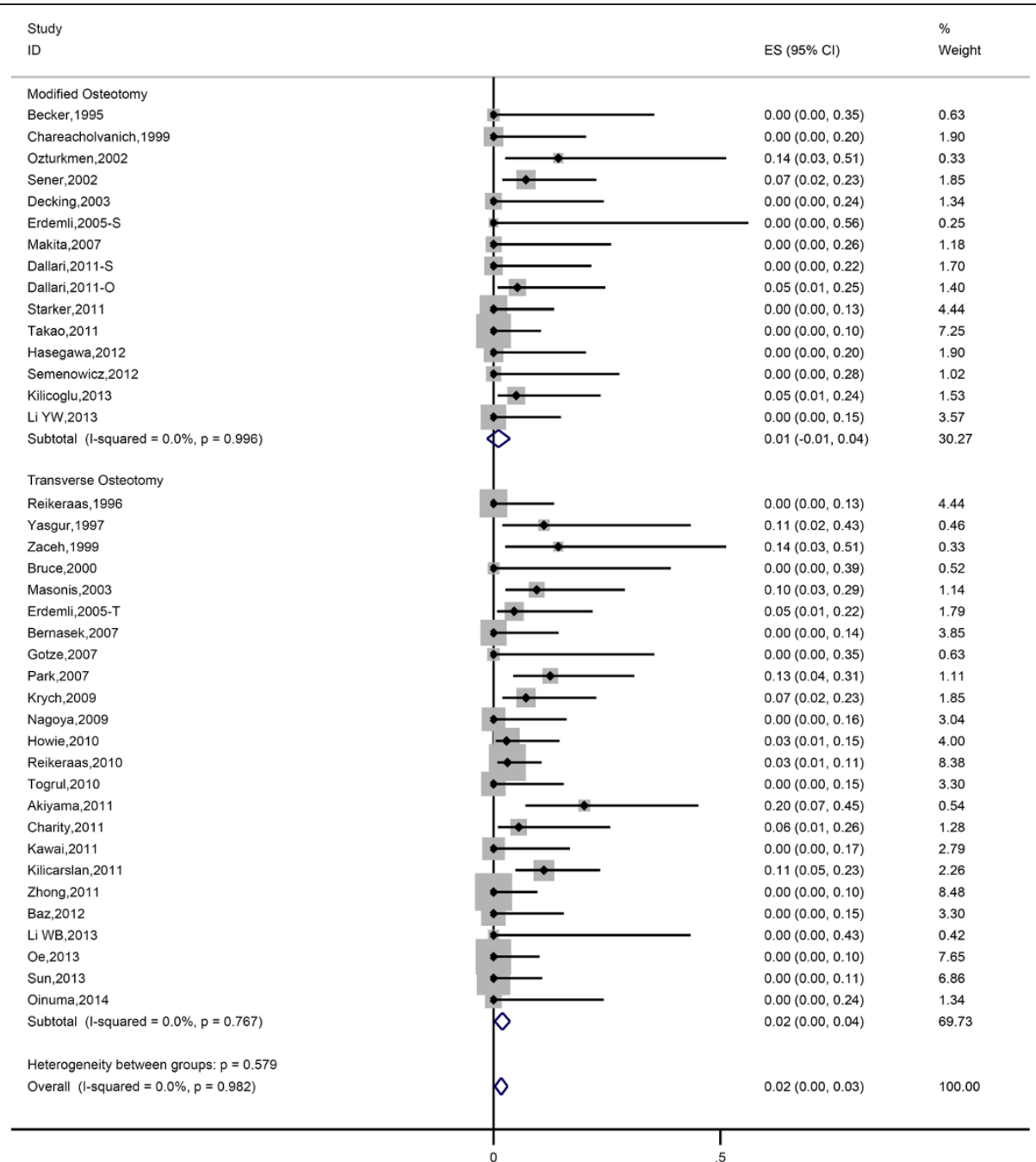

Figure 3 Forest plot of all eligible studies for nonunion rate.

solely responsible for the heterogeneity of any result. The results were almost the same as those when all studies were involved.

\section{Meta-regression}

Univariate meta-regression analysis was carried out for outcomes of interest to explore potential influencing factors. Method of osteotomy was found to be not associated with nonunion rate $(P=0.472)$, as well as other postoperative outcomes including nerve palsy $(P=0.240)$, dislocation $(P=0.735)$, revision $(P=0.653)$ and Harris hip score improvement $(P=0.562)$ (Table 3$)$.

Results of the meta-regression on revision rate indicated that western countries and longer follow-up ( $>5 y)$ are associated with higher revision rate (Table 3 ).

Meta-regression analysis was not performed for leglength discrepancy, due to limited quantity of eligible studies. Similarly, there was only one case of deep infection among all the involved studies, and thus metaregression was not performed for deep infection.

\section{Publication bias assessment}

Begg's funnel plots and Egger's regression asymmetry tests were performed to investigate whether publication bias existed (Figure 7). All outcomes were distributed symmetrically in funnel plots, indicating no obvious publication bias. Egger's regression asymmetry tests for nonunion, nerve palsy, revision, dislocation, leg-length discrepancy and HHS improvement showed no evidence of publication bias ( $P$ value was $0.380,0.186,0.714,0.165,0.524$ and 0.393 , respectively). $P$ value of Egger's test for deep infection was 0.044 , indicating a potential publication bias.

\section{Discussion}

This meta-analysis included 15 studies concerning modified osteotomy and 24 studies concerning transverse 
Table 2 Outcomes of meta-analysis and subgroup analysis

\begin{tabular}{|c|c|c|c|c|c|c|c|c|}
\hline \multirow[t]{2}{*}{ Outcome of interest } & \multirow{2}{*}{$\begin{array}{l}\text { No. of } \\
\text { studies }\end{array}$} & \multirow{2}{*}{$\begin{array}{l}\text { No. } \\
\text { of } \\
\text { hips }\end{array}$} & \multirow{2}{*}{$\begin{array}{l}\text { No. of } \\
\text { events }\end{array}$} & \multirow{2}{*}{$\begin{array}{l}\text { Pooled } \\
\text { estimate }\end{array}$} & \multirow[t]{2}{*}{$95 \% \mathrm{Cl}$} & \multicolumn{3}{|c|}{ Study heterogeneity } \\
\hline & & & & & & $x^{2}$ & $1^{2}$ & $P$ value \\
\hline Nonunion & 39 & 791 & 27 & $3.79 \%$ & $(2.60 \%, 5.20 \%)$ & 39.25 & $3.2 \%$ & 0.414 \\
\hline Modified subgroup & 15 & 241 & 5 & $3.03 \%$ & $(1.29 \%, 5.51 \%)$ & 8.29 & $0.0 \%$ & 0.874 \\
\hline Transverse subgroup & 24 & 550 & 22 & $4.14 \%$ & $(2.67 \%, 5.20 \%)$ & 30.34 & $24.2 \%$ & 0.140 \\
\hline Nerve palsy & 33 & 712 & 14 & $2.63 \%$ & $(1.60 \%, 3.87 \%)$ & 21.9 & $0.0 \%$ & 0.910 \\
\hline Dislocation & 27 & 641 & 38 & $5.88 \%$ & $(4.22 \%, 7.80 \%)$ & 42.35 & $36.2 \%$ & 0.030 \\
\hline Modified subgroup & 9 & 163 & 9 & $5.47 \%$ & $(2.57 \%, 9.36 \%)$ & 12.99 & $38.4 \%$ & 0.112 \\
\hline Transverse subgroup & 18 & 478 & 29 & $6.03 \%$ & $(4.14 \%, 8.29 \%)$ & 29.28 & $38.5 \%$ & 0.045 \\
\hline Revision & 22 & 482 & 45 & $8.90 \%$ & $(6.56 \%, 11.50 \%)$ & 45.57 & $53.9 \%$ & 0.001 \\
\hline Modified subgroup & 8 & 148 & 10 & $6.66 \%$ & $(3.31 \%, 11.12 \%)$ & 14.04 & $50.1 \%$ & 0.050 \\
\hline Transverse subgroup & 14 & 334 & 35 & $9.96 \%$ & $(7.06 \%, 13.35 \%)$ & 29.99 & $56.7 \%$ & 0.005 \\
\hline Limb-length discrepancy & 7 & $132^{*}$ & $10^{*}$ & $6.31 \%$ & $(2.90 \%, 11.00 \%)$ & 18.37 & $67.3 \%$ & 0.005 \\
\hline Modified subgroup & 4 & 72 & 8 & $9.02 \%$ & $(3.64 \%, 16.40 \%)$ & 13.86 & $78.4 \%$ & 0.003 \\
\hline Transverse subgroup & 3 & 60 & 2 & $3.72 \%$ & $(0.50 \%, 9.72 \%)$ & 2.83 & $29.3 \%$ & 0.243 \\
\hline HHS improvement & 17 & 337 & / & $26.79 \%$ & $(26.35 \%, 27.32 \%)$ & 654.87 & $97.6 \%$ & $<0.001$ \\
\hline Modified subgroup & 6 & 107 & / & $27.41 \%$ & $(26.52 \%, 28.31 \%)$ & 569.55 & $99.1 \%$ & $<0.001$ \\
\hline Transverse subgroup & 11 & 230 & / & $26.61 \%$ & $(25.99 \%, 27.14 \%)$ & 82.56 & $87.9 \%$ & $<0.001$ \\
\hline Deep infection & 19 & 393 & 1 & $1.34 \%$ & $(0.46 \%, 2.67 \%)$ & 2.24 & $0.0 \%$ & 1.000 \\
\hline
\end{tabular}

${ }^{*}$ Number of patients; Cl: confidential interval. 


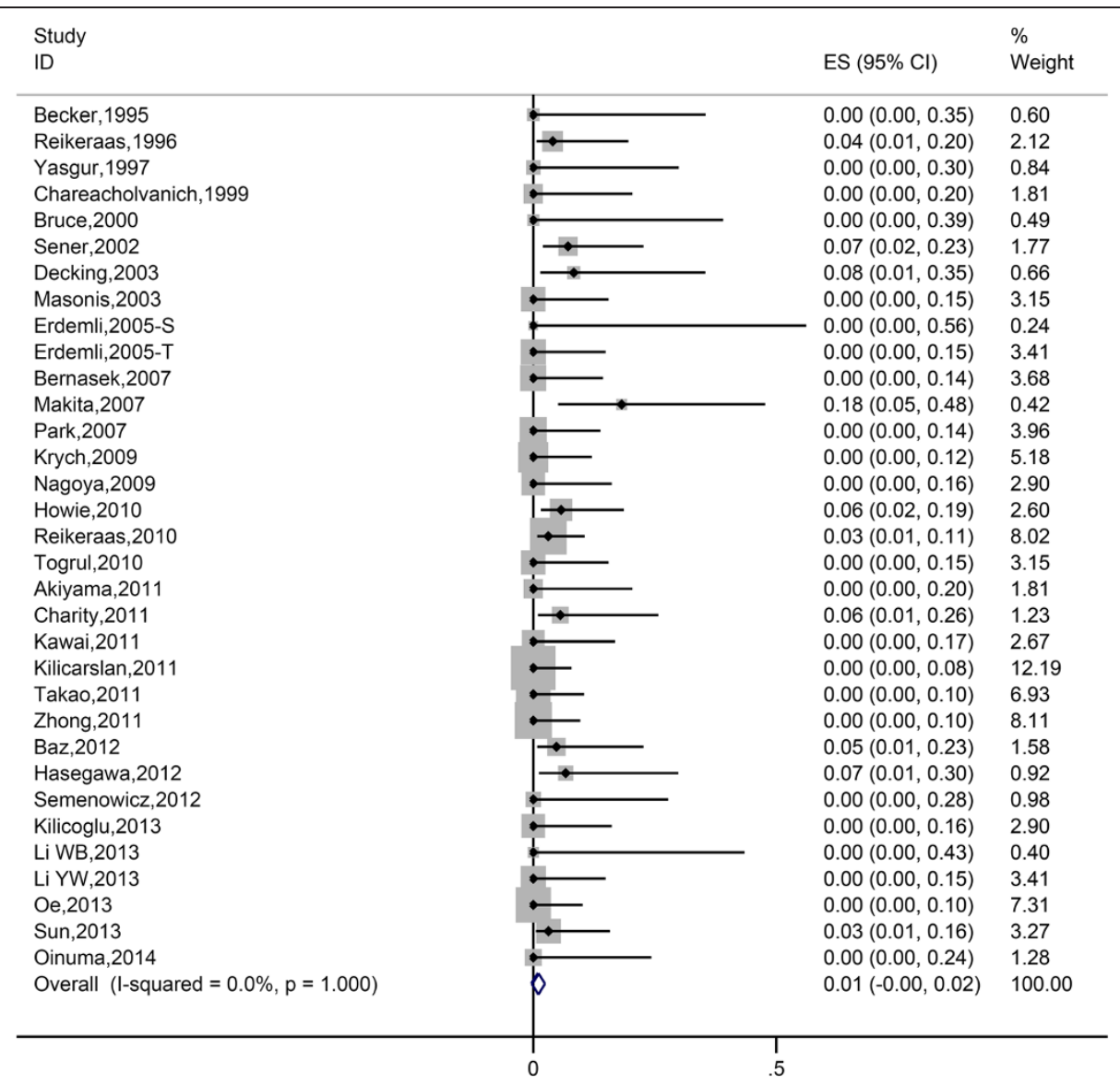

Figure 4 Forest plot of all eligible studies for nerve palsy rate.

osteotomy. The results showed that method of osteotomy was not associated with nonunion rate, as well as other post-operative outcomes including nerve palsy, dislocation, revision, leg-length discrepancy, HHS improvement and deep infection. In addition, western countries and longer follow-up $(>5.1 \mathrm{y})$ were associated with higher revision rate.

According to our analysis, transverse osteotomy and modified osteotomy didn't show significant difference in nonunion rate (Figure 3). The analysis also showed that transverse and modified osteotomy didn't show significant difference in terms of occurrence of nerve palsy, indicating that they have equal efficacy in preventing post-operative complication concerning nerve stretching (Figure 4). This could be attributed to the fact that the occurrence of nerve palsy is predominantly associated with the extent of leg lengthening.

In addition, occurrence of other post-operative complication including dislocation and deep infection is similar between transverse osteotomy and modified osteotomy. Recognized risk factors of dislocation after total hip arthroplasty include age, sex, head diameter of femoral prosthesis, surgical approach and experience of surgeon [52]. It wasn't unexpected that method of osteotomy had no influence on post-operative dislocation. As for the relationship between method of osteotomy and infection rate, there had been different opinions. Modified osteotomies seem to take more time than transverse osteotomies due to their complexity $[45,46,49]$, and there were several studies showing that prolonged operation time would increase the risk of deep infection after total hip arthroplasty [53-55]. The deduction may seem reasonable that modified osteotomy would have higher infection rate than transverse osteotomy. However, our meta-analysis revealed that modified osteotomy and transverse osteotomy shared similar infection rate. This is probably because that the difference of operation time for modified and transverse osteotomy was not so significant to bring a difference in infection rate. On the other hand, some studies suggested that operation time might have no significant impact on deep infection rate at all [56,57]. As for revision rate, there's also no significant difference. In term of clinical improvement, modified osteotomy didn't seem to show better performance than transverse osteotomy in postoperative leg-length discrepancy and HHS. 


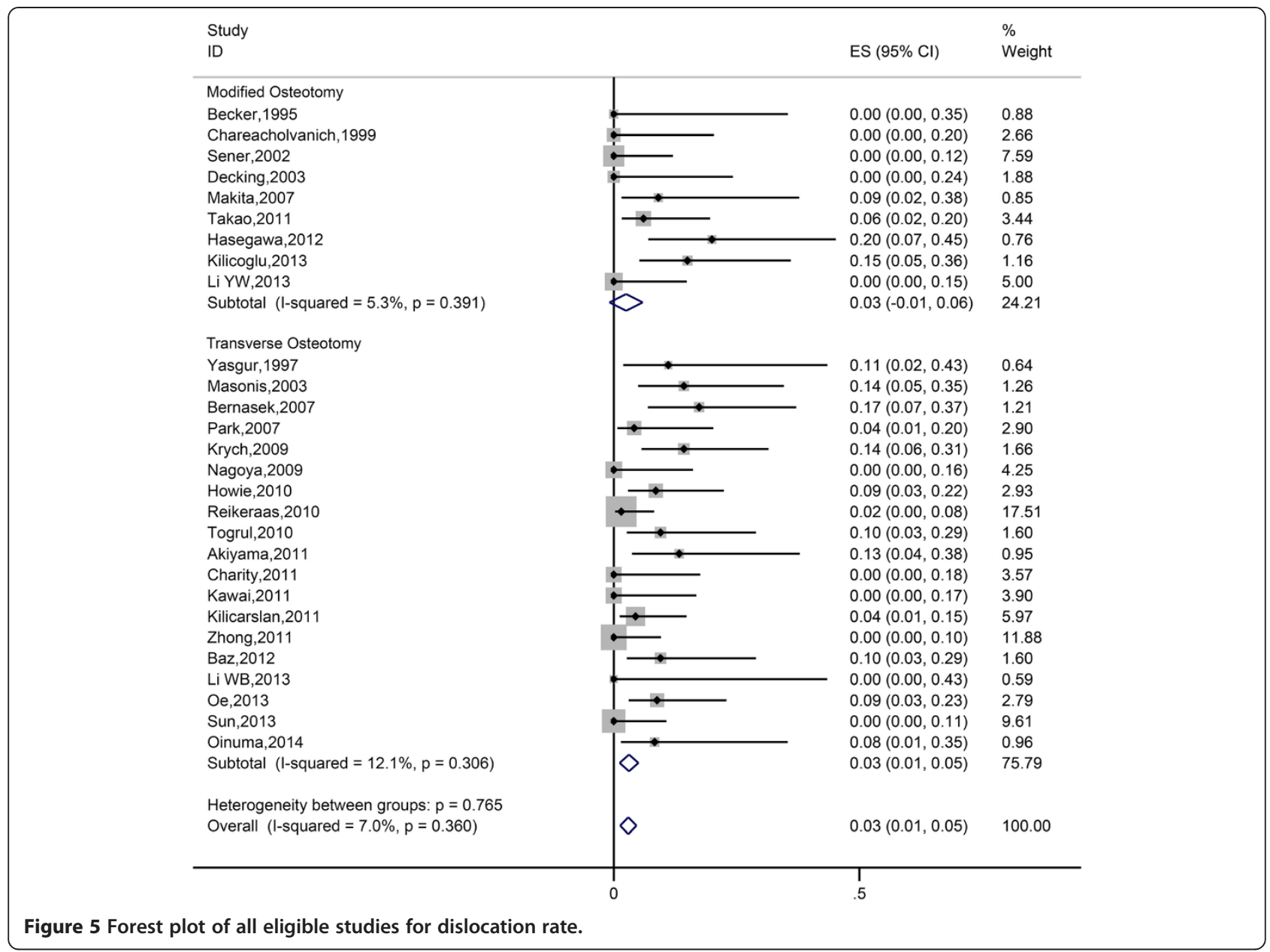

There was continuous suspicion that transverse osteotomy may not yield so good bone union as modified osteotomy, because of its lesser contact area for bone union, and potential rotational instability $[9,58]$. However, our analysis showed that transverse osteotomy could yield similar bone union as modified osteotomy. In the meantime, transverse osteotomy equally improved hip function, and is equally safe in avoiding complications as modified osteotomy. And modified osteotomy is reported to be under the risk of arm fracture of osteotomy as a postoperative complication [14], which is impossible after transverse osteotomy. We concluded that transverse osteotomy share similar nonunion rate with modified osteotomy, and is equally safe and effective as modified osteotomy.

As a matter of fact, derotation for femoral anteversion is usually necessary, which in turn requires prompt preoperative preparation and extreme accuracy of osteotomy to correct the femoral anteversion [13,17]. For modified osteotomy, once dissection is performed, there would be no chance for further adjustment of the anteversion angle of the femoral neck. Modified osteotomy also takes longer time than transverse osteotomy, due to the complexity of the surgical procedure, and the technically difficult procedure needs a lot of time and exercise for surgeons to master. Transverse osteotomy may be recommended, due to its simplicity and convenience in adjusting the anteversion angle.

Researchers have been trying to enhance the rotational stability of osteotomy, in order to improve bone union. Meanwhile, additional remarks should be made that rotational stability is influenced by not only the method of osteotomy, but also the design of implant used. Usually, DDH patient who receives THA is not so old to be free of risk of revision, and bone cement impedes later revision. On the other hand, possible leakage of bone cement would disturb the bone union. Taking these into consideration, researchers tended to perform cementless THA for DDH patients who needed subtrochanteric femoral shortening osteotomy at the same time. However, cross section and coating of femoral prostheses vary due to different designs. More importantly, press-fit femoral stem used in cementless THA mainly rely on proximal fixation, which might be insufficient to provide favorable stability for subtrochanteric osteotomy, for subtrochanteric 


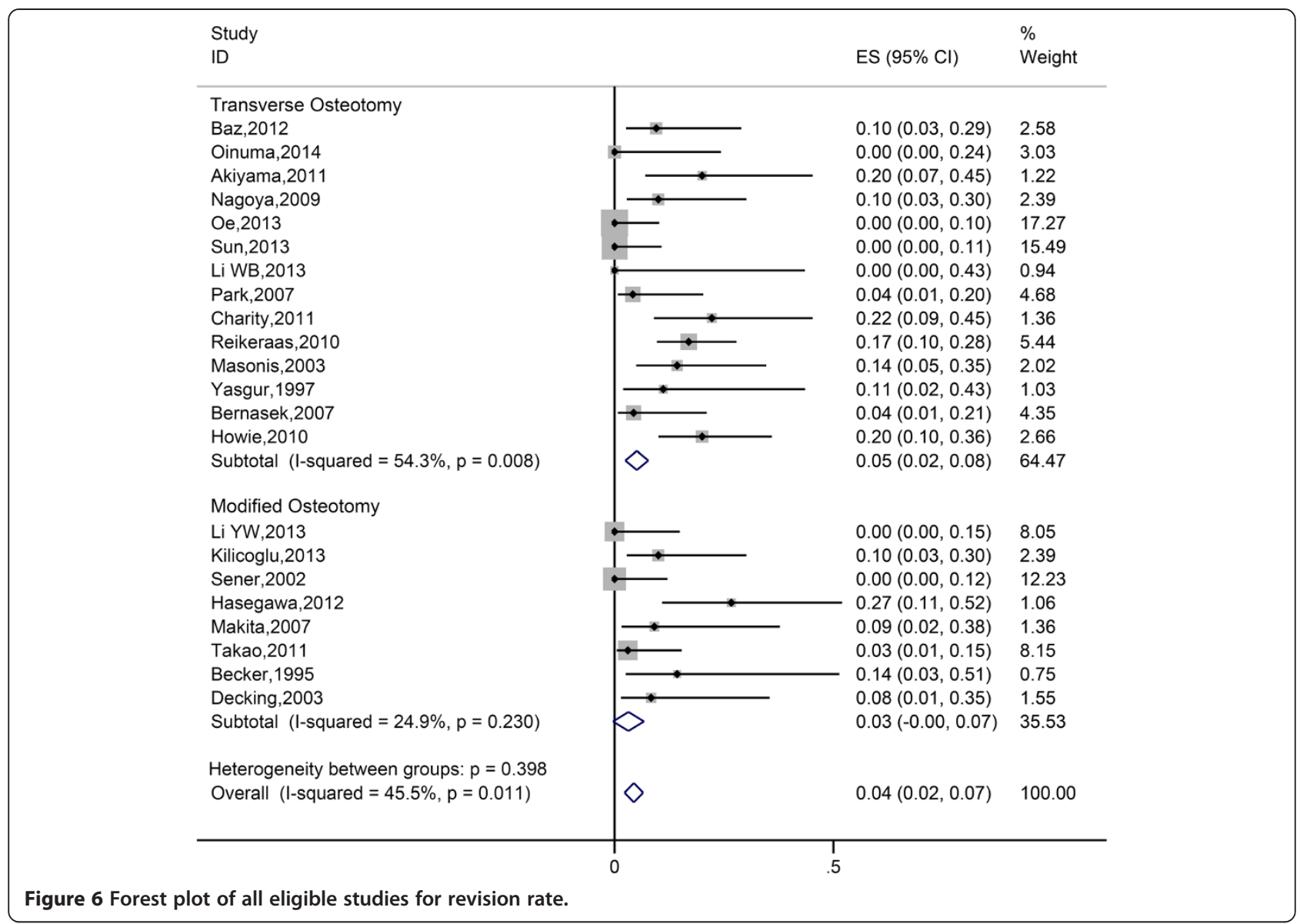

osteotomy itself requires stable fixation of both proximal and distal femur, on metaphysis and diaphysis, respectively [43]. In recent years, some researchers applied modular femoral stem (e.g. S-ROM, DePuy) to these patients $[33,34,43,44,46,48]$, and there were few reports on nonunion among them. Due to its distinctive design, modular stem guarantees both proximal and distal fixation, and thus has potential advantages in providing rotational stability for subtrochanteric osteotomy. Nevertheless, more studies are expected before consensus is achieved whether modular stem facilitates better union of subtrochanteric osteotomy.

Table 3 Outcomes of meta-regression analysis

\begin{tabular}{|c|c|c|c|c|c|c|c|c|c|c|}
\hline & \multicolumn{2}{|c|}{ Nonunion } & \multicolumn{2}{|c|}{ Nerve palsy } & \multicolumn{2}{|c|}{ Revision } & \multicolumn{2}{|c|}{ Dislocation } & \multicolumn{2}{|c|}{ HHS improvement } \\
\hline & $\bar{P}$ & Co. & $P$ & Co. & $\bar{P}$ & Co. & $P$ & Co. & $\bar{P}$ & Co. \\
\hline Method of osteotomy (modified vs. transverse) & 0.472 & -0.030 & 0.240 & 0.051 & 0.653 & -0.033 & 0.735 & -0.020 & 0.562 & -0.025 \\
\hline Country (western vs. eastern) & 0.731 & 0.013 & 0.272 & 0.042 & 0.010 & 0.169 & 0.592 & 0.029 & 0.819 & -0.010 \\
\hline Mean Age & 0.141 & -0.004 & 0.734 & 0.000 & 0.760 & -0.001 & 0.919 & 0.000 & 0.736 & -0.001 \\
\hline Mean Age (<49y vs. $\geq 49 y)$ & 0.302 & 0.042 & 0.958 & -0.002 & 0.980 & 0.002 & 0.948 & -0.004 & 0.940 & 0.004 \\
\hline Proportion of female & 0.845 & 0.000 & 0.587 & 0.000 & 0.721 & 0.001 & 0.367 & 0.003 & 0.290 & -0.002 \\
\hline Proportion of female (<93\% vs. $\geq 93 \%$ ) & 0.645 & 0.019 & 0.833 & -0.009 & 0.793 & 0.021 & 0.723 & 0.026 & 0.143 & 0.073 \\
\hline Follow up & 0.906 & 0.000 & 0.700 & 0.003 & 0.074 & 0.021 & 0.937 & 0.000 & 0.408 & -0.008 \\
\hline Follow up ( $\leq 5 \mathrm{y}$ vs. $>5 y$ ) & 0.937 & 0.003 & 0.871 & -0.006 & 0.014 & -0.163 & 0.391 & -0.046 & 0.354 & 0.042 \\
\hline Year of publication (2010 or before vs. after 2010) & 0.187 & 0.051 & 0.244 & 0.044 & 0.300 & 0.071 & 0.716 & 0.019 & 0.504 & 0.028 \\
\hline
\end{tabular}

HHS: Harris hip score; Co.: meta-regression coefficient. 


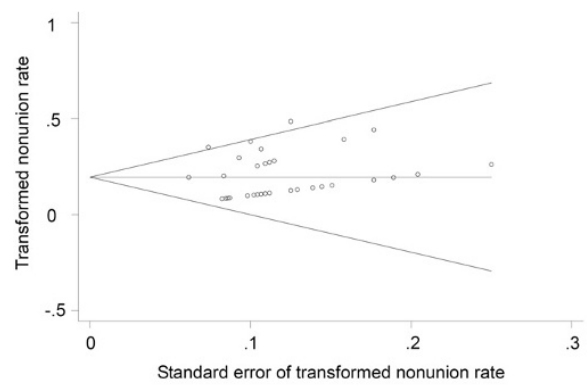

(a)

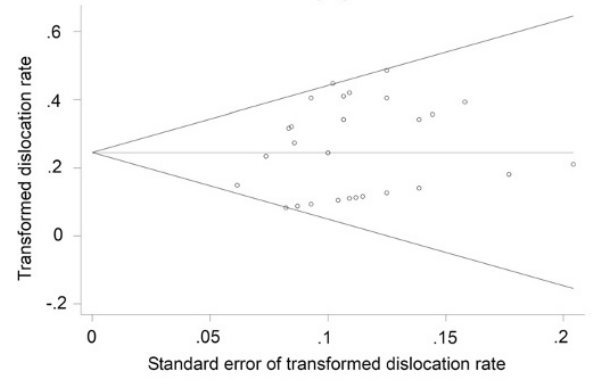

(c)

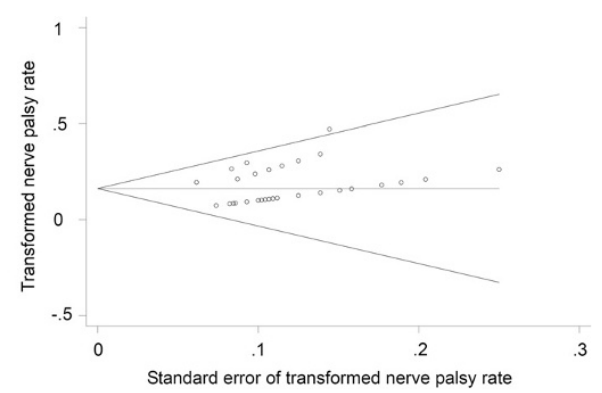

(b)

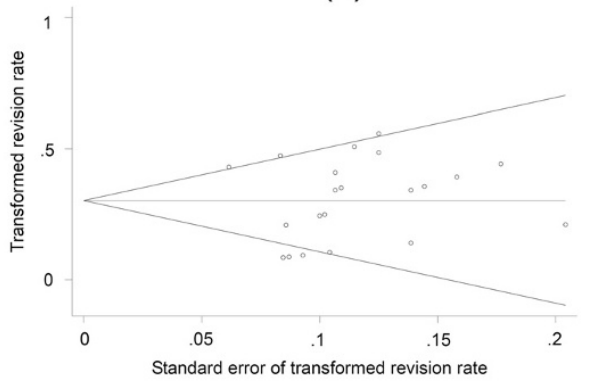

(d)

Figure 7 Begg's funnel plot of all eligible studies for (a) nonunion rate (b) nerve palsy (c) dislocation rate (d) revision rate.

Further analysis indicated that western countries and longer follow-up ( $>5 y)$ are associated with higher revision rate. Longer follow-up was to be expected on the basis of common sense and reasonable deduction. Western countries turned out to be associated with higher revision rate, probably due to its correlation with longer follow-up. Mean follow-up was 8.6 years in the studies of western countries, while 6.0 years in those of eastern countries.

This meta-analysis has the following limitations that must be taken into account. First, all the studies included were not randomized controlled trials, and thus of lower level of evidence. The studies were conducted with varying protocols and different levels of surgical expertise. Second, we compare transverse osteotomy and modified osteotomy, which is a category including stepcut, oblique and double-chevron osteotomy. There were much less studies on each of the modified osteotomy than on transverse osteotomy, which would result in significant loss of statistical efficacy if we compare each to transverse osteotomy separately. On the basis that the step-cut, oblique and double-chevron osteotomy shared some certain common ground, we categorized them into modified osteotomy in statistical analysis, as a strategy against potential loss of statistical efficacy. Third, the computer-based literature was systematic, and supplemented with manual searches. However, we may not be able to identify all the relevant studies despite our precise selection. In addition, the quantity of eligible studies was limited, and meta-regression analysis was not carried out for limb-length discrepancy due to lack of data.
To the best of our knowledge, this is the first metaanalysis comparing transverse and modified subtrochanteric femoral shortening osteotomy in THA for DDH patients. Second, it was conducted at an appropriate time, when enough data have accumulated for inspection by meta-analytical method. Third, this study was based on systematic up-to-date searching and filtering of literature, strictly according to the predetermined inclusion and exclusion criteria. Moreover, non-English language studies were included to minimize publication bias $[36,44,46,48,49]$. In addition, the variances of the raw rates were stabilized using Freeman-Tukey double-arcsine transformation, for the data were binary with low probability. Last but not least, it brings out a universal conclusion by including studies of various continents and countries. This meta-analysis therefore provides the most up-todate information in this area.

\section{Conclusions}

This meta-analysis indicated that transverse osteotomy shared similar nonunion rate with modified osteotomy, and was equally safe and effective as modified osteotomy. Transverse osteotomy may be recommended, due to its simplicity and convenience in adjusting the anteversion angle. Despite our rigorous methodology, the inherent limitations of the included studies are barriers for us to reach definitive conclusions. Well-designed and large-sample-size randomized controlled trials are expected to confirm and update the findings of this analysis. 


\section{Additional file}

\section{Additional file 1: A detailed description of the literature screening} and filtering.

\section{Competing interests}

The authors declare that they have no competing interests.

\section{Authors' contributions}

$\mathrm{CL}$ and $\mathrm{YD}$ participated in the design of this study, and they both performed the statistical analysis. CL and CZ collected important background information, and drafted the manuscript. MZ participated in the design of the study and data extraction and helped in the statistical analysis. All authors have read and approved the final manuscript.

\section{Acknowledgements}

This study was not supported by any company or grants.

Received: 11 June 2014 Accepted: 29 September 2014 Published: 3 October 2014

\section{References}

1. Dezateux C, Rosendahl K: Developmental dysplasia of the hip. Lancet 2007, 369:1541-1552

2. Peled E, Eidelman M, Katzman A, Bialik V: Neonatal incidence of hip dysplasia: ten years of experience. Clin Orthop Relat Res 2008, 466:771-775

3. Crowe JF, Mani VJ, Ranawat CS: Total hip replacement in congenital dislocation and dysplasia of the hip. J Bone Joint Surg Am 1979, 61:15-23.

4. Hartofilakidis G, Stamos K, Karachalios T, loannidis T, Zacharakis N: Congenital hip disease in adults. Classification of acetabular deficiencies and operative treatment with acetabuloplasty combined with total hip arthroplasty. J Bone Joint Surg Am 1996, 78:683-692.

5. Sanchez-Sotelo J, Berry DJ, Trousdale RT, Cabanela ME: Surgical treatment of developmental dysplasia of the hip in adults: II. Arthroplasty options. J Am Acad Orthop Surg 2002, 10:334-344.

6. Rosenstein $A D$, Diaz RJ: Challenges and solutions for total hip arthroplasty in treatment of patients with symptomatic sequelae of developmental dysplasia of the hip. Am J Orthop (Belle Mead NJ) 2011, 40:87-91.

7. Krych AJ, Howard JL, Trousdale RT, Cabanela ME, Berry DJ: Total hip arthroplasty with shortening subtrochanteric osteotomy in Crowe type-IV developmental dysplasia. J Bone Joint Surg Am 2009, 91:2213-2221.

8. Dunn HK, Hess WE: Total hip reconstruction in chronically dislocated hips. J Bone Joint Surg Am 1976, 58:838-845.

9. Kilicoglu Ol, Turker M, Akgul T, Yazicioglu O: Cementless total hip arthroplasty with modified oblique femoral shortening osteotomy in Crowe type IV congenital hip dislocation. J Arthroplasty 2013, 28:117-125.

10. Yang S, Cui Q: Total hip arthroplasty in developmental dysplasia of the hip: review of anatomy, techniques and outcomes. World J Orthop 2012, 3:42-48.

11. Zadeh HG, Hua J, Walker PS, Muirhead-Allwood SK: Uncemented total hip arthroplasty with subtrochanteric derotational osteotomy for severe femoral anteversion. J Arthroplasty 1999, 14:682-688.

12. Reikeras $\mathrm{O}$, Haaland JE, Lereim P: Femoral shortening in total hip arthroplasty for high developmental dysplasia of the hip. Clin Orthop Relat Res 2010, 468:1949-1955.

13. Baz AB, Senol $V$, Akalin $S$, Kose $O$, Guler $F$, Turan A: Treatment of high hip dislocation with a cementless stem combined with a shortening osteotomy. Arch Orthop Trauma Surg 2012, 132:1481-1486.

14. Dallari D, Pignatti G, Stagni C, Giavaresi G, Del Piccolo N, Rani N, Veronesi F Fini M: Total hip arthroplasty with shortening osteotomy in congenital major hip dislocation sequelae. Orthopedics 2011, 34:e328-e333.

15. Decking J, Decking R, Schoellner C, Fuerderer S, Rompe JD, Eckardt A: Cementless total hip replacement with subtrochanteric femoral shortening for severe developmental dysplasia of the hip. Arch Orthop Trauma Surg 2003, 123:357-362.

16. Becker DA, Gustilo RB: Double-chevron subtrochanteric shortening derotational femoral osteotomy combined with total hip arthroplasty for the treatment of complete congenital dislocation of the hip in the adult. Preliminary report and description of a new surgical technique. J Arthroplasty 1995, 10:313-318.
17. Makita H, Inaba Y, Hirakawa K, Saito T: Results on total hip arthroplasties with femoral shortening for Crowe's group IV dislocated hips. J Arthroplasty 2007, 22:32-38.

18. Strom L: Textbook of Pharmacoepidemiology. West Sussex: John Wiley \& Sons; 2007.

19. Moher D, Liberati A, Tetzlaff J, Altman DG, Group P: Preferred reporting items for systematic reviews and meta-analyses: the PRISMA statement BMJ 2009, 339:b2535.

20. Freeman M, Tukey J: Transformations related to the angular and the square root. Ann Math Stats 1950, 21:607-611.

21. Higgins JP, Thompson SG: Quantifying heterogeneity in a meta-analysis. Stat Med 2002, 21:1539-1558

22. Miller J: The inverse of the freeman-Tukey double arcsine transformation. Am Stat 1978, 32:138-138.

23. Higghins J, Green S: Cochrane Handbook for Systematic Reviews of Interventions; 2008

24. Reikeraas O, Lereim P, Gabor I, Gunderson R, Bjerkreim I: Femoral shortening in total arthroplasty for completely dislocated hips: 3-7 year results in 25 cases. Acta Orthop Scand 1996, 67:33-36.

25. Gotze C, Winkelmann W, Gosheger G, Rodl R: Is there a need of an additional extramedullary fixation in transverse subtrochanteric shortening in primary total hip arthroplasty for patients with severe hip dysplasia? Short-term experience in seven patients with congenital dislocation Z Orthop Unfall 2007, 145:568-573.

26. Howie CR, Ohly NE, Miller B: Cemented total hip arthroplasty with subtrochanteric osteotomy in dysplastic hips. Clin Orthop Relat Res 2010, 468:3240-3247.

27. Charity JA, Tsiridis E, Sheeraz A, Howell JR, Hubble MJ, Timperley AJ, Gie GA: Treatment of Crowe IV high hip dysplasia with total hip replacement using the Exeter stem and shortening derotational subtrochanteric osteotomy. J Bone Joint Surg Br 2011, 93:34-38.

28. Starker M, Bischof F, Lindenfeld T: Total hip arthroplasty with shortening subtrochanteric osteotomy and custom-made prosthesis in Crowe type IV developmental dysplasia. Z Orthop Unfall 2011, 149:518-525.

29. Semenowicz J, Szymanski S, Walo R, Czuma P, Pijet B: Total hip arthroplasty with shortening subtrochanteric $\mathbf{Z}$ osteotomy in the treatment of developmental dysplasia with high hip dislocation. Ortop Traumatol Rehabil 2012, 14:341-349.

30. Yasgur DJ, Stuchin SA, Adler EM, DiCesare PE: Subtrochanteric femoral shortening osteotomy in total hip arthroplasty for high-riding developmental dislocation of the hip. J Arthroplasty 1997, 12:880-888.

31. Chareancholvanich K, Becker DA, Gustilo RB: Treatment of congenital dislocated hip by arthroplasty with femoral shortening. Clin Orthop Relat Res 1999, 360:127-135.

32. Masonis JL, Patel JV, Miu A, Bourne RB, McCalden R, Macdonald SJ, Rorabeck $\mathrm{CH}$ : Subtrochanteric shortening and derotational osteotomy in primary total hip arthroplasty for patients with severe hip dysplasia: 5-year follow-up. J Arthroplasty 2003, 18:68-73.

33. Bernasek TL, Haidukewych GJ, Gustke KA, Hill O, Levering M: Total hip arthroplasty requiring subtrochanteric osteotomy for developmental hip dysplasia: 5- to 14-year results. J Arthroplasty 2007, 22:145-150.

34. Bruce WJ, Rizkallah SM, Kwon YM, Goldberg JA, Walsh WR: A new technique of subtrochanteric shortening in total hip arthroplasty: surgical technique and results of 9 cases. J Arthroplasty 2000, 15:617-626.

35. Erdemli B, Yilmaz C, Atalar H, Guzel B, Cetin I: Total hip arthroplasty in developmental high dislocation of the hip. J Arthroplasty 2005 20:1021-1028.

36. Ozturkmen Y, Karli M, Dogrul C: Cemented total hip arthroplasty for severe dysplasia or congenital dislocation of the hip. Acta Orthop Traumatol Turc 2002, 36:195-202.

37. Sener N, Tozun IR, Asik M: Femoral shortening and cementless arthroplasty in high congenital dislocation of the hip. J Arthroplasty 2002, 17:41-48.

38. Park MS, Kim KH, Jeong WC: Transverse subtrochanteric shortening osteotomy in primary total hip arthroplasty for patients with severe hip developmental dysplasia. J Arthroplasty 2007, 22:1031-1036.

39. Nagoya S, Kaya M, Sasaki M, Tateda K, Kosukegawa I, Yamashita T: Cementless total hip replacement with subtrochanteric femoral shortening for severe developmental dysplasia of the hip. J Bone Joint Surg Br 2009, 91:1142-1147. 
40. Togrul E, Ozkan C, Kalaci A, Gulsen M: A new technique of subtrochanteric shortening in total hip replacement for Crowe type 3 to 4 dysplasia of the hip. J Arthroplasty 2010, 25:465-470.

41. Akiyama H, Kawanabe K, Yamamoto K, Kuroda Y, So K, Goto K, Nakamura T: Cemented total hip arthroplasty with subtrochanteric femoral shortening transverse osteotomy for severely dislocated hips: outcome with a 3- to 10-year follow-up period. J Orthop Sci 2011, 16:270-277.

42. Kilicarslan K, Yalcin N, Karatas F, Catma F, Yildirim H: Cementless total hip arthroplasty for dysplastic and dislocated hips. Eklem Hastalik Cerrahisi 2011, 22:8-15.

43. Takao M, Ohzono K, Nishii T, Miki H, Nakamura N, Sugano N: Cementless modular total hip arthroplasty with subtrochanteric shortening osteotomy for hips with developmental dysplasia. J Bone Joint Surg Am 2011, 93:548-555.

44. Zhong C, Cai XZ, Yan SG, He RX: S-ROM modular arthroplasty combined with transverse subtrochanteric shortening for Crowe type IV congenital dislocation of hip. Chin Med J (Engl) 2011, 124:3891-3895.

45. Hasegawa Y, Iwase T, Kanoh T, Seki T, Matsuoka A: Total hip arthroplasty for Crowe type developmental dysplasia. J Arthroplasty 2012, 27:1629-1635.

46. Sun QC, Wang XH, Song BS, Zhu FB, Yan SG: Total hip arthroplasty for crowe type IV developmental dysplasia of the hip with S-ROM prosthesis. Zhongguo Gu Shang 2013, 26:153-157.

47. Kawai T, Tanaka C, Ikenaga M, Kanoe H: Cemented total hip arthroplasty with transverse subtrochanteric shortening osteotomy for Crowe group IV dislocated hip. J Arthroplasty 2011, 26:229-235.

48. Li W, Zhang W, Bai G, Huang Z, Shen R: Total hip arthroplasty for treatment of Crowe type IV congenital dysplasia of hip with dislocation in adults. Zhongguo Xiu Fu Chong Jian Wai Ke Za Zhi 2013, 27:1153-1156.

49. Li Y, Ma W, Sun J, Song X, An M, Zhang Q: Becker V-shaped lateral rotation osteotomy in total hip arthroplasty for Crowe type IV development dislocation of hip. Zhongguo Xiu Fu Chong Jian Wai Ke Za Zhi 2013, 27:1032-1036.

50. Oe K, lida H, Nakamura T, Okamoto N, Wada T: Subtrochanteric shortening osteotomy combined with cemented total hip arthroplasty for Crowe group IV hips. Arch Orthop Trauma Surg 2013, 133:1763-1770.

51. Oinuma K, Tamaki T, Miura Y, Kaneyama R, Shiratsuchi H: Total hip arthroplasty with subtrochanteric shortening osteotomy for Crowe grade 4 dysplasia using the direct anterior approach. J Arthroplasty 2014, 29:626-629.

52. Brooks PJ: Dislocation following total hip replacement: causes and cures. Bone Joint J 2013, 95-B:67-69.

53. Jamsen $\mathrm{E}$, Varonen M, Huhtala H, Lehto MU, Lumio J, Konttinen YT, Moilanen T: Incidence of prosthetic joint infections after primary knee arthroplasty. J Arthroplasty 2010, 25:87-92.

54. Dale H, Hallan G, Hallan G, Espehaug B, Havelin LI, Engesaeter LB: Increasing risk of revision due to deep infection after hip arthroplasty. Acta Orthop 2009, 80:639-645.

55. Pulido L, Ghanem E, Joshi A, Purtill JJ, Parvizi J: Periprosthetic joint infection: the incidence, timing, and predisposing factors. Clin Orthop Relat Res 2008, 466:1710-1715.

56. Harrison T, Robinson P, Cook A, Parker MJ: Factors affecting the incidence of deep wound infection after hip fracture surgery. J Bone Joint Surg Br 2012, 94:237-240.

57. Edwards C, Counsell A, Boulton C, Moran CG: Early infection after hip fracture surgery: risk factors, costs and outcome. J Bone Joint Surg $B$ 2008, 90:770-777

58. Bracken J, Tran T, Ditchfield M: Developmental dysplasia of the hip: controversies and current concepts. J Paediatr Child Health 2012, 48:963-972. quiz 972-963.

doi:10.1186/1471-2474-15-331

Cite this article as: Li et al:: Comparison of transverse and modified subtrochanteric femoral shortening osteotomy in total hip arthroplasty for developmental dysplasia of hip: a meta-analysis. BMC Musculoskeletal Disorders 2014 15:331.

\section{Submit your next manuscript to BioMed Central and take full advantage of:}

- Convenient online submission

- Thorough peer review

- No space constraints or color figure charges

- Immediate publication on acceptance

- Inclusion in PubMed, CAS, Scopus and Google Scholar

- Research which is freely available for redistribution

Submit your manuscript at www.biomedcentral.com/submit 